\title{
Optical Design of a Reflecting Telescope for CubeSat
}

\author{
Ho Jin $^{1}$, Juhee Lim $^{2}$, Youngju Kim ${ }^{3 *}$, and Sanghyuk Kim ${ }^{1}$ \\ ${ }^{1}$ School of Space Research, Kyung Hee University, YongIn 446-701, Korea \\ ${ }^{2}$ Satellite Technology Research Center, KAIST, Daejeon 305-701, Korea \\ ${ }^{3}$ Yunam Optics, Inc., Icheon 467-811, Korea
}

(Received August 21, 2013 : revised October 23, 2013 : accepted October 24, 2013)

\begin{abstract}
Space telescope optics is one of the major parts of any space mission used to observe astronomical targets or the Earth. This kind of space mission typically involves bulky and complex opto-mechanics with a long optical tube, but attempts have been made to observe a target with a small satellite. In this paper, we describe the optical design of a reflecting telescope for use in a CubeSat mission. For this design we adopt the off-axis segmented method for astronomical observation techniques based on a Ritchey-Chrétien type telescope. The primary mirror shape is a rectangle with dimensions of $8 \mathrm{~cm} \times 8$ $\mathrm{cm}$, and the secondary mirror has dimensions of $2.4 \mathrm{~cm} \times 4.1 \mathrm{~cm}$. The focal ratio is 3 which can yield a 0.383 degree diagonal angle in a $1280 \times 800 \mathrm{CMOS}$ color image sensor with a pixel size of $3 \mu \mathrm{m}$ $\times 3 \mu \mathrm{m}$. This optical design can capture $\mathrm{a} \sim 4 \mathrm{~km} \times \sim 2.3 \mathrm{~km}$ area of the earth's surface at $700 \mathrm{~km}$ altitude operation.
\end{abstract}

Keywords: Telescope, Optical design, Space optics

OCIS codes : (220.4830) Optical systems design; (350.6090) Space Optics; (110.6770) Telescopes

\section{INTRODUCTION}

A telescope for space exploration requires numerous types of technology. In particular, the imaging system is one of the most essential components for scientific research. Generally, astronomical space telescopes and satellite cameras use a reflecting optical system with conic mirrors because the conic surface can be very precise, and the radiation tolerance is much higher than that of refracting lens material $[1$, 2]. There are many types of space optics with one or two mirrors used for low-resolution imaging or spectroscopic observation [3, 4]. Recent imaging sensor technology also allows us to design a wider field of view and achieve higher resolution in space imaging with various satellite platforms. In this paper, we present the optical design of a small reflecting telescope for a $3 \mathrm{U}$ CubeSat platform.

CubeSat is a class of nano-satellites initially developed at Stanford University and California Polytechnic State University at San Louis Obispo to facilitate low-cost access to space [5]. The CubeSat platform is a standardized configuration, consisting of one to three $10 \mathrm{~cm} \times 10 \mathrm{~cm} \times 10$ $\mathrm{cm}$ units $(1,2$, or 3 "U"s) arranged in a row, where $1 \mathrm{U}$ has a mass of $1.33 \mathrm{~kg}[6,7]$. It is launched as a piggyback secondary payload on a large launch vehicle using the Poly-Picosatellite Orbital Deployer (P-POD). There are many kinds of $3 \mathrm{U}$ CubeSat missions for scientific research and technical experiments $[8,9]$. Imaging satellites based on CubeSat have mostly been implemented for Earthobserving missions.

Most imaging CubeSats have refraction lens mount optics because it is difficult to fit a reflecting telescope into a CubeSat. Lens mount optics has disadvantages such as the darkening effect caused by space radiation and, misalignment and fragility caused by vibration and thermal effects.

In this paper, we design the optics of a reflecting telescope for a CubeSat unlike previous missions. A major objective is to acquire high-quality images of the Earth's surface with our new, small reflecting telescope.

\section{BASIC REQUIREMENTS FOR THE CUBESAT TELESCOPE}

\subsection{Concept}

This research involves the design of a conventional

\footnotetext{
*Corresponding author: smallisle@yunamoptics.com

Color versions of one or more of the figures in this paper are available online.
} 


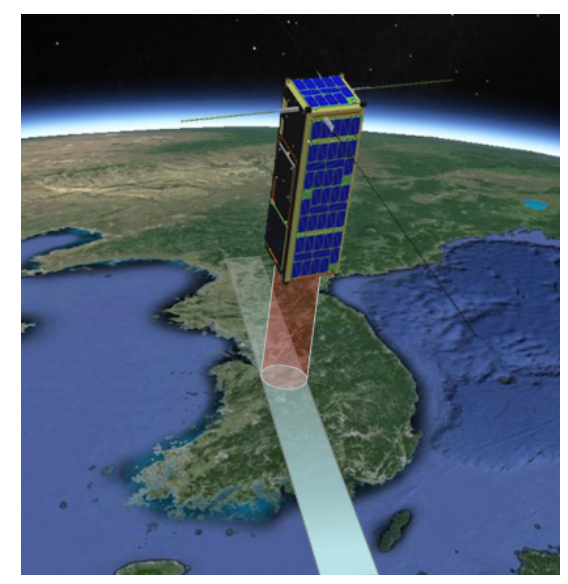

FIG. 1. Concept of CubeSat mission operation to collect images of Earth.

imaging camera system with a small reflecting telescope for a CubeSat mission. We estimate a $\sim 700 \mathrm{~km}$ altitude in sun synchronous orbit with a standard CubeSat platform. Figure 1 shows the conceptual operation of the pushbroom imaging mode to scan or shoot the Earth's surface as the CubeSat moves along its orbit. In this section, we describe the fundamental requirements for designing the reflecting telescope for a $3 \mathrm{U}$ CubeSat mission $(10 \mathrm{~cm} \times$ $10 \mathrm{~cm} \times 34 \mathrm{~cm})$.

\subsection{Mechanical Design Requirements}

The main constraint of the optical design is the space allowance in CubeSat. There should be no interference in the optical path within the limited CubeSat dimensions. The CubeSat should have avionic electronics and basic structures. Therefore, the telescope space is limited by these constraints. In this design, the minimum space for avionics electronics is $10 \mathrm{~cm} \times 10 \mathrm{~cm} \times 10 \mathrm{~cm}$, and other structural parts require $10 \mathrm{~cm} \times 10 \mathrm{~cm} \times 5 \mathrm{~cm}$. Therefore, the maximum allowed space for the telescope is $10 \mathrm{~cm} \times$ $10 \mathrm{~cm} \times 19 \mathrm{~cm}$. However, the telescope optics should be installed within a space of $8 \mathrm{~cm} \times 8 \mathrm{~cm} \times 19 \mathrm{~cm}$ in order to allow for installation of a support structure for the telescope optics and satellite structure. For this reason, we configured the optics within a space of $8 \mathrm{~cm} \times 8 \mathrm{~cm} \times 19$ $\mathrm{cm}$, as shown in Fig. 2.

\subsection{Optical Design Concept}

The main purpose of this telescope optics system is a fine resolution image of Earth's surface with the CubeSat application. In the case of the CubeSat mission, we can easily find an imaging instrument with a refraction lens mount within this platform because reflecting telescope optics is difficult to fit into a tiny space [7]. In this paper, we design a reflecting telescope optics system unlike the refraction lens design.

The telescope design parameters take into account several

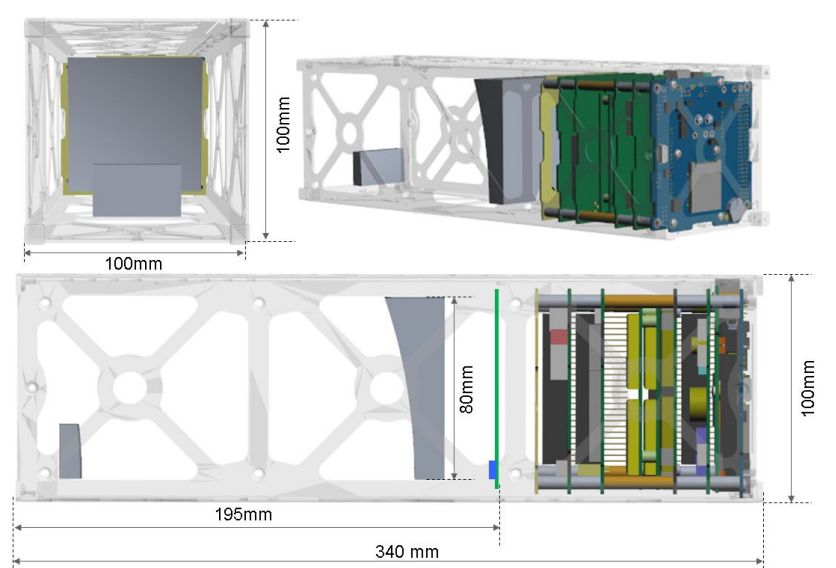

FIG. 2. Optical configuration of the CubeSat structure $(8 \mathrm{~cm} \times$ $8 \mathrm{~cm} \times 19 \mathrm{~cm})$.

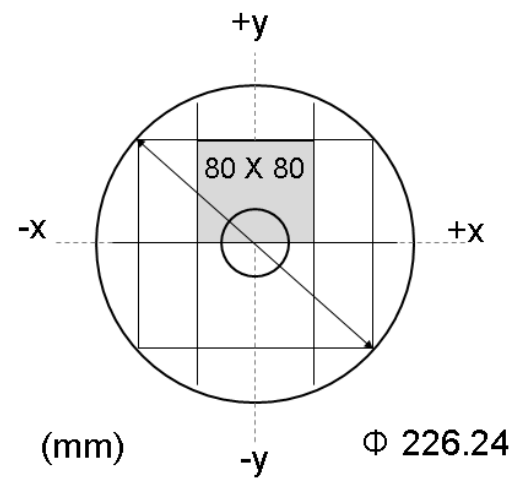

FIG. 3. Aperture mask concept. Hatched rectangle is the primary mirror.

volume limitations. Two-mirror optics is a primary consideration; hence, a short focal length is also prioritized. In this case, the dimensions of the primary mirror must be within $8 \mathrm{~cm}$ $\times 8 \mathrm{~cm}$, to fit into the CubeSat envelope. The field of view (FOV) is small in two-mirror optical telescopes; for example, the FOV for the Ritchey-Chretien optical system, which has the largest FOV, is only a few minutes [10]. Therefore, we aim to increase the diameter to achieve a larger FOV compared to that in the Ritchey-Chretien system. Thus, the primary mirror shape becomes a square, which cuts off the edges from a round mirror, as shown in Fig. 3. The idea is based on the off-axis segmented technique in astronomical telescope applications. We set the focal image plane area to $5 \mathrm{~mm}$ diameter because the CMOS image sensing area cannot exceed $4.5 \mathrm{~mm}$. Table 1 shows the optical design parameters for our design. In this table we estimate the ground resolving distance based on the geometrical resolution, without diffraction. In the optical design process the diffraction effect should be of concern, since the detectors can be chosen scientifically so that more energy is collected each instant by every pixel in the FOV. [11]. 


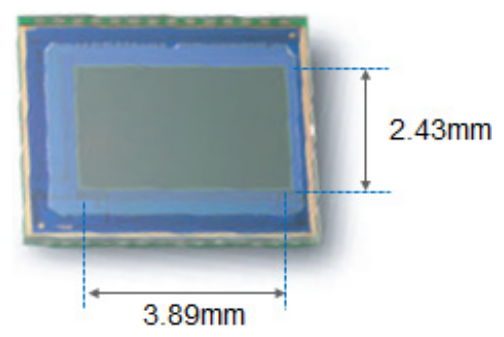

FIG. 4. Dimensions of the OV9712 CMOS image sensor.

TABLE 1. Design specifications of the telescope

\begin{tabular}{c|c}
\hline \hline Parameters & Specifications \\
\hline M1-M2 distance & $150 \pm 10 \mathrm{~mm}$ \\
\hline M1 thickness & $5 \mathrm{~mm}$ \\
\hline Image plane size & $5 \mathrm{~mm}$ diameter \\
\hline Field of View & $<0.7^{\circ}$ \\
\hline Ground Resolving Distance & $<10 \mathrm{~m}$ at $700 \mathrm{~km}$ \\
\hline Diffraction limit & $8.36 \mu \mathrm{m}$ \\
\hline Telescope type & Ritchey-Chrétien \\
\hline
\end{tabular}

\subsection{Image Sensor}

There are many kinds of sensors used to acquire an image. A CCD device is commonly used for astronomical observations due to its many advantages, such as a large full-well capacity, $100 \%$ fill factor, and low readout noise compared to a CMOS image sensor. In this study, however, we choose a CMOS image sensor because it has various pixel sizes and windowing readout mode, and low power consumption, unlike the CCD image sensor. In particular, windowing readout mode is very useful for Earth observation, to overcome the image blur produced by the satellite sweep speed during exposure. Our observation target is the day-time Earth surface, which is bright enough to allow collection of images with CMOS image sensors in the designed telescope. For our optical design, we choose the CMOS image sensor OV9712 produced by Omnivision. The pixel size is $3 \mu \mathrm{m} \times 3 \mu \mathrm{m}$ and the pixel array is $1280 \times 800$. Table 1 describes the CMOS image sensor used in our design.

\section{OPTICAL DESIGN AND ANALYSIS}

\subsection{Telescope Design}

The telescope is a conventional two-mirror telescope. We used part of a $226.24 \mathrm{~mm}$ diameter mirror as the telescope's primary mirror. It is a modified off-axis segmented method, as in astronomical observation. The primary and secondary mirrors are rectangular with dimensions of $80 \mathrm{~mm}$ $\times 80 \mathrm{~mm}$ and $41 \mathrm{~mm} \times 24 \mathrm{~mm}$, respectively. The rectangular shape maximizes captured light in the rectangular CubeSat body. Figure 5 shows the design layout in which the focal
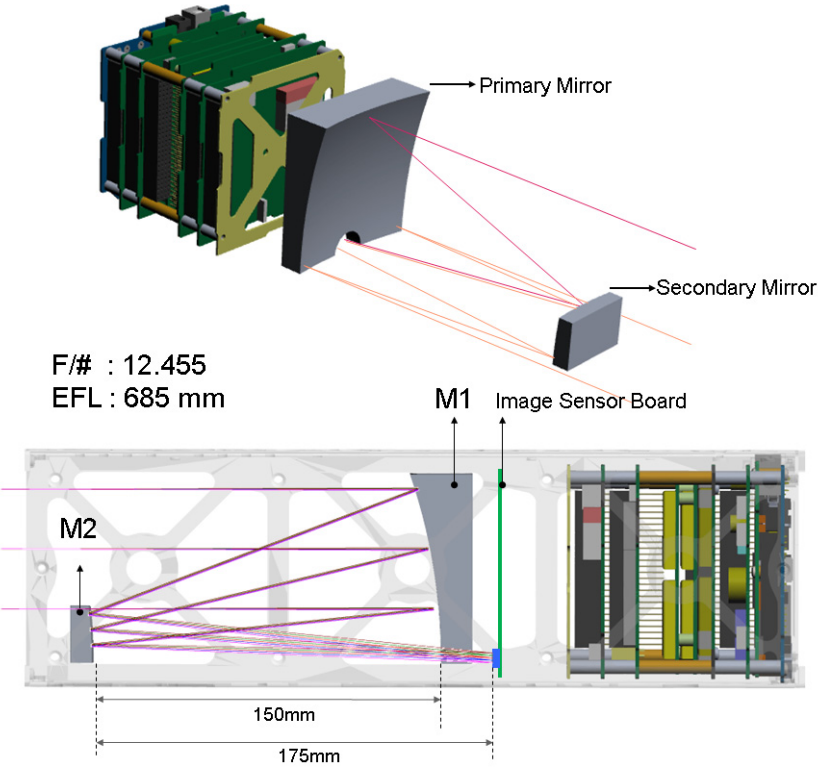

FIG. 5. Optical design layout of the CubeSat telescope.

TABLE 2. Imaging sensor specifications

\begin{tabular}{c|c}
\hline \hline Items & Specifications \\
\hline Active array size & $1280 \times 800$ \\
\hline Pixel size & $3 \mu \mathrm{m} \times 3 \mu \mathrm{m}$ \\
\hline Temperature range & $-30{ }^{\circ} \mathrm{C}$ to $70{ }^{\circ} \mathrm{C}$ \\
\hline Image area & $3.888 \mathrm{~mm} \times 2.430 \mathrm{~mm}$ \\
\hline Package dimension & $5415 \mu \mathrm{m} \times 4415 \mu \mathrm{m}$ \\
\hline
\end{tabular}

plane is located behind the primary mirror. Table 3 shows the data sheet for the optical design.

\subsection{Optical Performance Analysis}

The analysis was carried out with the Code- $\mathrm{V}$ optical design program. Figure 6 shows a spot diagram that includes the size and shape of the spot for all visible wavelengths. The spot size indicates that the outermost field $\left(0,0.094^{\circ}\right)$ and optical axis are $2.3 \mu \mathrm{m}$ and $3.3 \mu \mathrm{m}$, respectively.

This size is slightly larger than 2 pixels. We also investigated astigmatism and distortion; the field curve is presented in Fig. 7. The distortion is no greater than 2 pixels in scale around the full field, and there is no chromatic aberration due to the reflecting mirror system. We identified the astigmatism from the MTF diagram as shown in Fig. 8, where the solid line is the MTF value of the radial component of the curve, and the dashed line is the tangential component of the curve. The camera resolution is represented by Eq. (1) in the color image array sensor.

$$
\text { Camera Res }(l p / m m)=\frac{\# \text { Pixels }}{2 \times \text { Sensor size }}=\frac{1280 / 2}{2 \times 3.89}=82.26 \mathrm{lp} / \mathrm{mm}
$$


TABLE 3. Lens data sheet

\begin{tabular}{|c|c|c|c|c|c|c|}
\hline Surface \# & Surface Name & $\begin{array}{c}\text { Radius } \\
\text { (Conic constant) }\end{array}$ & $\begin{array}{l}\text { Thickness } \\
(\mathrm{mm})\end{array}$ & Refract Mode & Semi-Aperture (mm) & \\
\hline Object & - & - & - & - & - & \\
\hline Stop & - & - & 170 & - & - & $\begin{array}{l}\text { Y-axis decenter } \\
-100 \mathrm{~mm}\end{array}$ \\
\hline \multirow{2}{*}{2} & M1 & \multirow{2}{*}{$\begin{array}{c}-402.0036 \\
(\mathrm{k}=-1.0678)\end{array}$} & \multirow{2}{*}{-150} & \multirow{2}{*}{ Reflect } & $40 \times 40$ & \multirow{2}{*}{$\begin{array}{c}\mathrm{Y} \text {-axis decenter } \\
-50 \mathrm{~mm}\end{array}$} \\
\hline & Center hole & & & & 10 (semi-circle) & \\
\hline 3 & M2 & $\begin{array}{c}-145.9288 \\
(\mathrm{k}=-4.1274)\end{array}$ & 150 & Reflect & $24.5 \times 11$ & \\
\hline 4 & M1 thickness & - & 5 & - & - & \\
\hline 5 & - & - & 20 & - & - & \\
\hline Image & Sensor & - & - & - & 2.2491 & \\
\hline
\end{tabular}

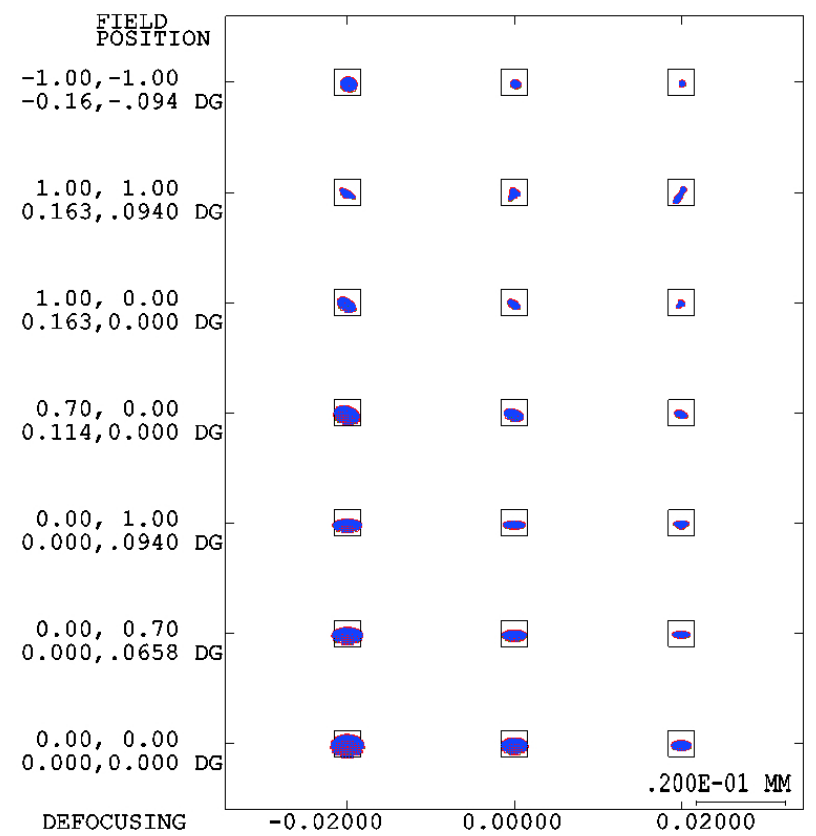

FIG. 6. Spot diagram. Square box is $6 \mu \mathrm{m} \times 6 \mu \mathrm{m}$.

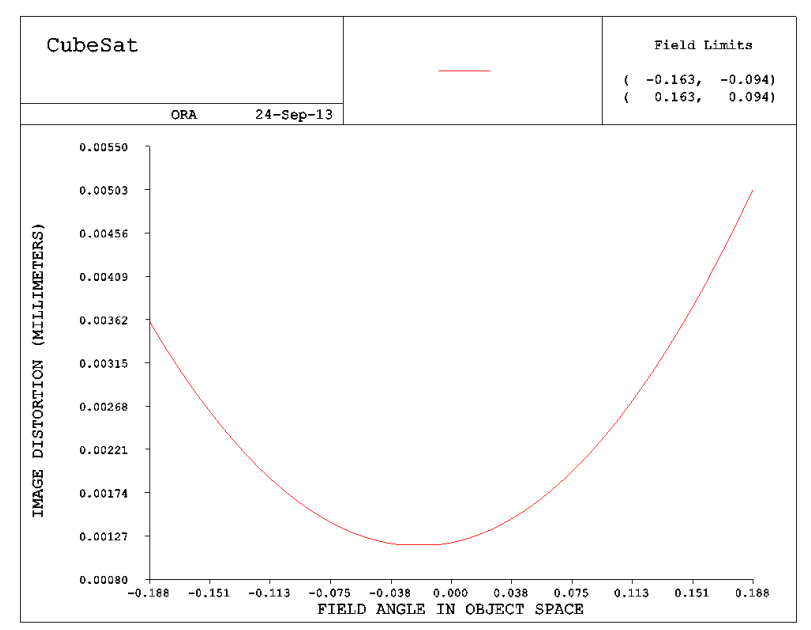

FIG. 7. Field curves.

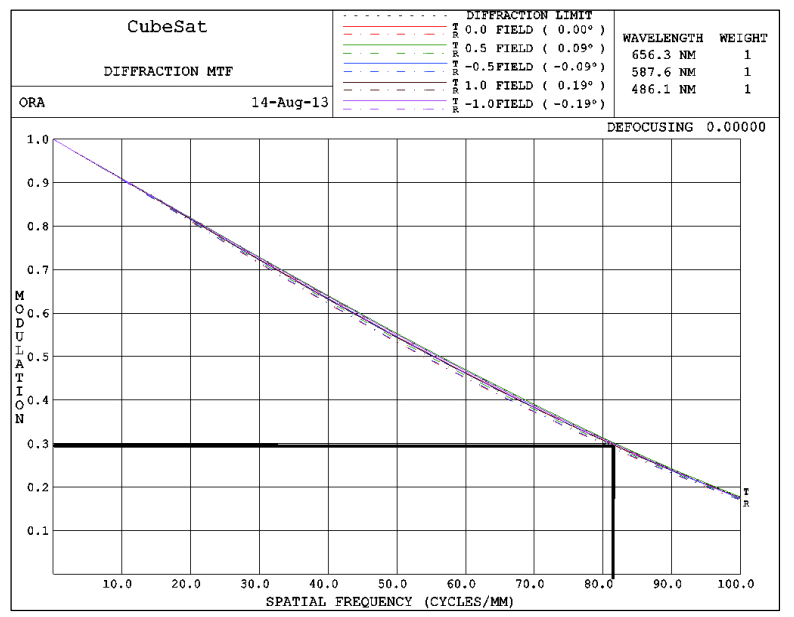

FIG. 8. Diffraction MTF.

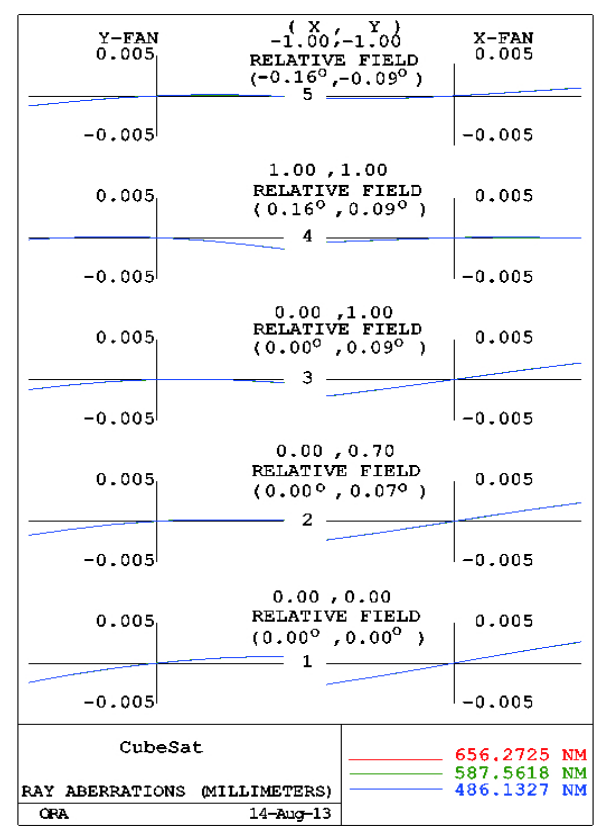

FIG. 9. Ray aberration plot. 
We observe that the minimum MTF value appears over 0.3 at the $82.26 \mathrm{lp} / \mathrm{mm}$. This is the diffraction MTF. In this case, the tangential and sagittal MTF are a little bit different. On the other hand, this design shows a small amount of fifth-order coma and large-angle astigmatism, and presents a field curvature effect that is relatively much larger than others, as shown in Fig. 9.

\section{CONCLUSION}

In this paper we present the optical design of a small reflecting telescope for a CubeSat application. This research considers issues pertaining to the assembly and manufacture of the rectangular mirror, which is designed within a space limitation. We chose a reflecting mirror to obtain high resolution with a small focal length and EPD in a 3U CubeSat.

A recent CMOS image sensor with tiny pixel size and large array also enables us to design the reflecting telescope in this study. It uses a Ritchey-Chrétien optical system, and its MTF is expected to be about 0.3 with a $3.2 \mathrm{~mm} \times$ $2.8 \mathrm{~mm}$ image area. The results show that theoretically it can take images of a $\sim 4 \mathrm{~km} \times \sim 2.3 \mathrm{~km}$ area of the Earth's surface at $700 \mathrm{~km}$ altitude. This telescope shows a reasonable design to obtain a more detailed image with a CubeSat. These results will be used in space exploration with CubeSat missions.

\section{ACKNOWLEDGMENT}

This study was partially supported by a grant from the KASI NAP program and also partially supported by the BK21 plus program through the National Research Foundation (NRF) funded by the Ministry of Education of Korea.

\section{REFERENCES}

1. G. I. Lebedeva and A. A. Garbul, "Prospective aerospace reflective objectives," J. Opt. Technol. 61, 610-614 (1994).

2. M. Fruit, A. I. Gusarov, D. B. Doyle, and G. J. Ulbrich, "Radiation impact on space borne optics, the dose coefficient approach," Proc. SPIE 3872, 60-71 (1999).

3. J. U. Lee and S. M. Yu, "Analytic design procedure of three-mirror telescope corrected for spherical aberration, coma, astigmatism, and Petzval field curvature," J. Opt. Soc. Korea 13, 184-192 (2009).

4. J. H. Lee, T. S. Jang, H.-S. Yang, and S.-W. Rhee, "Optical design of a compact imaging spectrometer for STSAT3,” J. Opt. Soc. Korea 12, 262-268 (2008).

5. A. Toorian, K. Diaz, and S. Lee, "The CubeSat approach to space access," in Proc. the IEEE Aerospace Conference (Big Sky, MT, USA, 2008), pp. 1-14.

6. H. Heidt, J. Puig-Suari, A. S. Moore, S. Nakasuka, and R. J. Twiggs, "CubeSat: A new generation of Picosatellite for education and industry low-cost space experimentation," in Proc. the Utah State University Small Satellite Conference (Logan, UT, USA, Aug. 2001), pp. 1-19.

7. M. W. Smith, S. Seager, C. M. Pong, J. S. Villaseñor, G. R. Ricker, D. W. Miller, M. E. Knapp, G. T. Farmer, and R. Jensen-Clem, "ExoplanetSat: Detecting transiting exoplanets using a low-cost CubeSat platform," Proc. SPIE 7731, 773127 (2010).

8. J. G. Yoo, H. Jin, J. H. Seon, Y. H. Jeong, D. Glaser, D. H. Lee, and R. P. Lin, "Thermal analysis of TRIO-CINEMA mission," Journal of Astronomy and Space Sciences 29, 23-31 (2012).

9. N. Y. Yoon, S. Y. Yoon, Y. H. Kim, J. W. Yoon, H. Jin, J. H. Seon, K. S. Chae, D. H. Lee, and R. P. Lin, "Development of CINEMA mission uplink communication system," Journal of Astronomy and Space Sciences 29, 33-40 (2012).

10. J. H. Pan, The Design, Manufacture and Test of the Aspheric Optical Surface (Science Press, Beijing, China, 1994), pp. 10-47.

11. X. Li, M. Xu, X. Ren, and Y. Pei, "An optical design off-axis four-mirror-anastigmatic telescope for remote sensing," J. Opt. Soc. Korea 16, 243-246 (2012). 